\title{
Improved Optimization Algorithm of Ant Colony
}

\author{
Zhao Yun-hong \\ College of Computer Engineering, Jingchu University of Technology, 448000, China
}

\begin{abstract}
The mechanisms and basic principles about ant colony algorithm is researched, and in system point of view such characteristics as positive feedback, self-organizing systems, and distributed computing of the ant colony algorithm are analyzed. Analysis, verify and classify improved optimization algorithm of ant colony in detail by TSP-Ei151 in MATLAB 7.6; It shows this algorithm superior to $\mathrm{AS}$ in convergence, global and number of iterations.
\end{abstract}

Key words- Optimization algorithm, convergence, improve operator

\section{I . Introduction}

Ant colony optimization algorithm ${ }^{[1,2,3]}$, which began in the last century 90 's, can be compared with the genetic algorithm which is established by the simulation of human evolution. It was first proposed by Italy scholar M.Dorigo etc. in 1991, it is a kind of evolutionary algorithm of bionic structure of simulation of ant foraging to seek the shortest path behavior. The inspiration is the social synergy that imitates the ants to communicate with the pheromone which guide the action of each ant, which is a self-organizing process guided by the greedy method.

The essence of ant colony foraging behavior is a kind of distributed collaborative optimization mechanism. It is almost impossible for individual ant to find the shortest path from nest to food source, but it is so easy for many ants to do that, the collective behavior of the ant colony composed of many ants highlights the wisdom of the ants, namely the ability to find the shortest path. Because ants have no sight, the process of communication in movement is indirect, that is, an ant releases a certain amount of information element on the path it passes through, other ants move where there is many more and more pheromone by the existence and strength of perception. The 'intelligent' behavior of ants is also reflected in the adaptability to the environment. When ants forage and encounter obstacles, they can avoid obstacles and find the optimal path to meet the constraint conditions in a shorter time. This kind of indirect communication between individual, to change environment and perception of environment, is called social synergy optimization mechanism.

Since the ant colony optimization algorithm succeed in solving the traveling salesman problem (TSP), it has achieved good application results to solve the vehicle scheduling, quadratic assignment, job shop scheduling problem, graph coloring problem, integrated circuit design and communication network in load problem ${ }^{[4]}$.

\section{II . Principle of ant colony algorithm}

M. Dorigo has been aware that ant system (ant system, AS) searching optimization process is slow and it is easy to stagnate, through continuous improvement, he in 1997 proposed ant colony system (ant colony system, ACS) ${ }^{[2]}$, this is one of the best performance of the ant colony optimization algorithm. Here I have solving the problem of symmetric TSP as an example of the principle of ant colony algorithm to do an introduction.

In order to simplify, first introduce some notation: the number of ants as $\sigma$, serial number of city as $n$, distance of city r to city s as $d_{r s}$, pheromone left by the ant between city $\mathrm{r}$ and s as $\tau_{r s}$.

The initial situation, pheromone on each path is equal, noted as $\tau_{0}$, select randomly some ants, noted as $\sigma$,some city of ants setting out, noted n.. Using 
repeatedly the state transition rule, make a construction of the path of each ant. At the same time, according to the local pheromone updating rules, update pheromone. If all ants have completed the construction of the path, the pheromone is updated according to the global pheromone updating rules. The flow chart of ant colony system is shown in Figure 1:

2.1 State transition rule

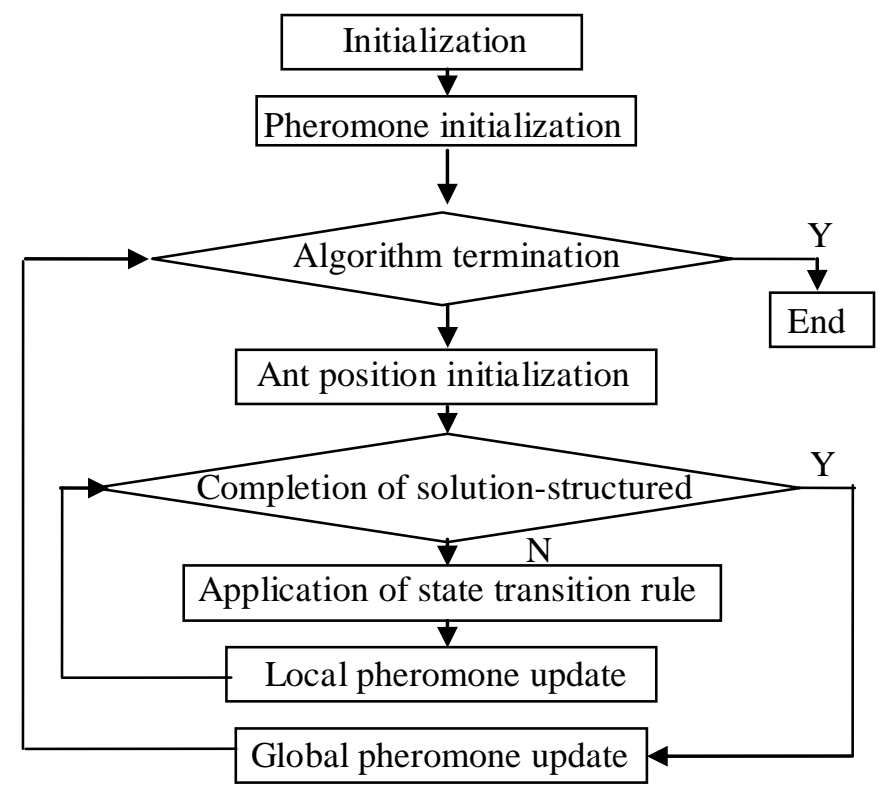

Figure 1 the flow chart of ant colony system

Suppose the current city which the ant k stays is $r$, and then according to the formula 1 and the rule of probability formula 2 to select the next city s:

$$
\begin{aligned}
& S=\left\{\begin{array}{l}
\arg _{u \in \text { allowed }_{k}}^{\max }\left\{[\tau(\gamma, \mu)]^{\alpha} \cdot[\eta(\gamma, \mu)]^{\beta}\right\}, \text { if } . q \leq q_{0} \\
\text { s, otherwise. }(1)
\end{array}\right. \\
& P_{i j}^{k}(t)=\left\{\begin{array}{l}
\frac{\tau_{i j}^{\alpha}(t) \cdot \eta_{i j}^{\beta}(t)}{\sum_{s \in \text { allowed }_{k}} \tau_{i s}^{\alpha}(t) \cdot \eta_{s j}^{\beta}(t)}, j \in \text { allowed }_{k} \\
\text { O, } \text { otherwise }
\end{array}\right.
\end{aligned}
$$

In above formula, $q \in$ random $[0,1], q_{0} \in[0,1]$

they are initial parameter. $P_{i j}^{k}(t)$ is the probability that ant $\kappa$ chooses city $s, \eta_{i j}(t)$ is the visibility of the ant for line $(i, j)$ at time $t$, as a heuristic information, $\beta$ is heuristic information weight. $\alpha$ is pheromone volatile parameter $(0<\alpha<1)$. The rule is called pseudo random equilibrium rule, In the search for the balance of the high quality solution and the search space, it can be carried out by choosing the value of the parameter $q_{0}$

\section{2 Local pheromone updating rules}

Between the nodes, if the ants move, then update the local pheromone according to the formula 3 :

$$
\tau_{r s}(t+1) \leftarrow \tau_{r s}(t) \rho+\rho \Delta \tau_{r s} .
$$

In the above formula, $\rho$ describes local evaporation coefficient of pheromone $(1<\rho<1)$, in general, the value of $\Delta \tau_{r s}$ is $\tau_{0}$. The role of this rule is to increase the diversity of search.

\subsection{Global pheromone updating rules}


When all of the ants have completed an ergodic, the global pheromone updating is based on Formula 4 and 5:

$$
\begin{gathered}
\tau_{r s}(t+1) \leftarrow(1-\alpha) \tau_{r s}(t)+\alpha \Delta \tau_{r s} . \\
\nabla \tau(r, s)=\left\{\begin{array}{l}
\frac{1}{L_{g b}}, \text { if }(r, s) \in \text { global }- \text { most }- \text { path }(5) \\
0, \text { otherwise. }
\end{array}\right.
\end{gathered}
$$

$\mathrm{L}_{\mathrm{gb}}$ is the length of the global optimal ergodic.

\section{Improved ant colony algorithm}

The ant colony algorithm proposed firstly by Dorigo M. etc. has slow convergence rate and premature convergence and low accuracy problem, he himself and other researchers made some improvements from mechanism, introducing operator and restricting candidate set etc.

\section{1 Improved mechanism of algorithm itself}

In the ant colony system (ACS), Dorigo M. introduced the pseudo random proportional rule (PRPR) to have the relationship to the balance between mining and exploration; in order to increase the diversity of the search, local pheromone updating rules are introduced; in order to enhance pheromone which ant passed by the optimal path and accelerate the convergence, the global pheromone updating rules are defined. In order to avoid search stagnation problem, German scholars Thomastuzle and Holerhoos introduced pheromone smoothing mechanism which constraints the range of pheromone on the path, this is max min ant system (MMAS). According to the distribution uniformity of optimization something about balancing the relationship between preventing premature convergence and accelerate the convergence, the adaptive ant colony algorithm based on distribution uniformity degree is proposed in literature [5], which can adaptively adjust the information pheromone updating strategy and route to choice probability. From the view of establishment of information pheromone diffusion model, based on better collaborate between near ants, the new ant colony algorithm which simulates real ant colony system is proposed in literature [6], which effectively improve the performance of the algorithm. A kind of TSP algorithm is mainly proposed in literature $[7,8]$, in the solution of subsection or meeting, two ants together search for a path, so that the search speed can be improved. Another ant colony algorithm is proposed in literature [9], its effectiveness and robustness is stronger, it is based on pheromone limited classification, information pheromone is updated through classes updating, and the pheromone updating quantity does not depend on the objective function value. The authors in literature [9] proposed a kind of ant colony algorithm based on pheromone concentration, in order to improve the efficiency of the search space; the thinking is the introduction of centralized mechanism which adjusts information elements according to its proportion. In addition, it increases selection probability for the low information pigment solution component.

3. 2 The idea of introducing operators or other algorithms

Austria's Heimer B. Bull, who introduced the concept of ordering to update the pheromone, proposed an ant system based on permutation (ant system rank-base, AS). Spanish scholars cordon o introduced evolutionary computing ideas to the ant colony optimization, he proposed best worst ant system (Best-Worst ant system, BWAS), increased the best and worst pheromone updating rule to significantly speed up the convergence, established pheromone variation and restart mechanism to avoid search stagnation. In literature $[11,12,13,14]$ mutation and crossover operator is introduced into the ant colony optimization to improve the diversity of the search. A new ant colony system, which contains the reconnaissance subgroup, is proposed in the paper[15], which is inspired by the thought of the reconnaissance troops of the human war. The algorithm is from ant colony isolated from an army of ants scouting subgroup, the subgroup randomly search in the optimization process with a certain probability, in order to improve the diversity of the search, results of TSP simulation experiment verify the algorithm greatly accelerate the convergence speed but also overcomes the 
premature phenomenon.

3. 3 Theory based on nearest neighbor candidate set

Candidate set theory is also an important strategy, in order to improve the convergence speed of the algorithm, which can limit the search scope of ants in a reduced search space. This is the most commonly used nearest neighbor candidate set. In literature $[2,3,16]$ candidate set are 15, 20 or a Windows of nearest neighbor ranging, the nearest neighbor based on $\mathrm{n} / \omega$ in literature [13] has as the candidate set. ( $\mathrm{n}$ is the number of cities in the traveling salesman problem (TSP), Omega is a variable parameter, $n / \omega$ epsilon $[5,20])$. And another improved method are proposed in a lot of other literature: some parameters are adjusted adaptively, some rules of local search algorithm are added, some random agitation mechanism is of introduction, some the information dynamic update..., limited to space, no longer lists. To a certain extent, these improvements have improved the speed of the algorithm and the quality of the solution. However, for large-scale combinatorial optimization problems such as large-scale TSP problems, it is too long for the solution and easy to fall into the local optimum, which is still worth discussing.

\section{Experimental results and its analysis}

Because of the natural relationship between the ant colony optimization algorithm and the TSP problem, its birth, even to all the improvements, and also present of the new algorithm mostly have TSP problem as a test case.

The basic ant colony algorithm and the improved algorithm are applied to the TSPlib-ei151 problem, the simulation experiment (experimental environment: Intel Core 2 P7370@2.00GHz/MATLAB7.6), the results are compared with table I and table II.

From the experimental data of two tables, we can see that the improved algorithm is obviously better than the basic algorithm: the number of iterations is small, the convergence and the overall situation is improved. it is an effective optimization algorithm.

Table I experimental results of different parameters of the basic algorithm

\begin{tabular}{c|c|c|c|c}
\hline$\alpha$ & $\beta$ & $\rho$ & Shortest path length & $\begin{array}{c}\text { Evolutionary algebra required for } \\
\text { convergence }\end{array}$ \\
\hline 2 & 4 & 0.93 & 433.2 & 324 \\
\hline 2 & 4 & 0.8 & 434.1 & 300 \\
\hline 4 & 2 & 0.9 & 440.1 & 341 \\
\hline 4 & 2 & 0.6 & 431.6 & 336 \\
\hline
\end{tabular}

Table II experimental results of different parameters of the improved algorithm

\begin{tabular}{c|c|c|c|c}
\hline$\alpha$ & $\beta$ & $\rho_{\text {min }}$ & Shortest path length & Evolutionary algebra required for convergence \\
\hline 2 & 4 & 0.1 & 424.9 & 236 \\
\hline 2 & 4 & 0.5 & 425.6 & 248 \\
\hline 4 & 2 & 0.5 & 426.6 & 240 \\
\hline 4 & 2 & 0.1 & 424.9 & 234 \\
\hline
\end{tabular}

\section{Conclusion}

A comprehensive analysis and classification of the improved ant colony algorithm is carried out. Research on bionic evolution such as ant colony algorithm is a kind of effective method to solve the complex problem. Indeed, it is not a long time for the study of ant colony algorithm, most achievements remain just in the analysis of a large number of experimental data, and many still in 
the simulation stage, there is no solid mathematical foundation and it is relatively complex to selection of various parameters, therefore, from the view of improving the algorithm theory there are many urgent problems. With the development of the theory and the further research, the application of ant colony algorithm will be more and more extensive.

\section{Reference}

[1] Colormi A,Dorgo M,Manieaao V.Distributed Optimization by Ant Colonies.Varela $F$ and Bourgine P.Proc of the First European Conf On Artificial Life[C].Paris,France:Elsevier Publishing, 1991.134-142.

[2] Dorigo M,Gambardella L M.Ant Colony System:A Cooperative Learning Approach to the Traveling Salesman Problem.IEEE Trans on Evolutionary Computation[J],1997,1(1):53-66.

[3] Dorigo $M$ and Di Caro GThe Ant Colony Optimization Meta-heuristic.Corne D,Dorigo M,Glover F.New Ideas in Optimization[M].London, UK,McGraw-Hill,1999.1 $1-32$.

[4] Duan Haibing. Ant colony algorithm principle and its application [M]. Beijing: Science Press. 2007.

[5] Chen Leng,Shen Jie,Qin Lin, Chen Hong jian. An adaptive ant colony algorithm based on distributed uniformity. Software Journal [J], 2003, 14 (8): 1379-1387.

[6] Hang Guo rui,Cao Xian bin, Wang Xi fa. Ant colony algorithm based on pheromone diffusion. Journal of electronics [J], 2004, 32 (5): 865-868.

[7] Wu bing,Shi Zhong zhi. An ant colony algorithm based on TSP problem segmentation algorithm. Journal of computers [J], 2001, 24 (12): 1328-1333.

[8] Zhao Wen bing,Sun Zhi yi,Li Hong. An ant colony algorithm for solving the TSP problem. Computer Engineering [J], 2004, 30 (12): 136-137185.

[9] Ke Liang jun,Feng $\mathrm{Zu}$ ren,Feng Yuan jing. Finite pheromone ant colony algorithm. Journal of automation [J], 2006,32 (2): 296-303.

[10] Song Zheng, Guangxing Zhang and Zekui Zhou.Ant Colony Optimization based on Pheromone Trail Centraliztiion. Proceedings of the 6th Word Congress on Intelligent Control and Automation[C], Dalian, China, 2006.3349-3352.

[11] Wu Qing hong, Zhang Jihui,Xu Xin he. Ant colony algorithm with variable features. Computer research and development [J], 1999, 36 (10): 1240-1245.

[12] Chen Ye. Ant colony algorithm with crossover operator. Computer Engineering [J], 2001, 27 (12): 74-76176.

[13] Zhu Qing Bao,Yang Zhi jun. Ant colony optimization algorithm based on mutation and dynamic pheromone updating. Journal of software [J], 2004, 15 (2): 185-192.

[14] Zhu Hai mei, Zhu Qing bao,Hu Yong Ant colony algorithm with adaptive hybrid characteristics. Computer engineering and applications [J], 2004, 40 (22): 81-83, 96.

[15] Xu Jian,Lv Zhi min,Xu Jin wu. Ant colony system with scout groups. Journal of University of Science and Technology Beijing [J], 2006, 28 (8): 794-798.

[16] Xiao Yunsi,Li Bingyu. Small window ant colony algorithm. Computer Engineering [J], 2003, 29 (20): 143-145. 